\title{
Shape Variation-Based Frieze Pattern for Robust Gait Recognition
}

\author{
Seungkyu Lee* \\ skleeepsu.edu \\ Yanxi Liu*† \\ Robert Collins ${ }^{\dagger}$ \\ rcollinsacse.psu.edu \\ $\dagger$ Dept. of Computer Science and Eng. *Dept. of Electrical Eng. \\ The Penn State University
}

\begin{abstract}
Gait is an attractive biometric for vision-based human identification. Previous work on existing public data sets has shown that shape cues yield improved recognition rates compared to pure motion cues. However, shape cues are fragile to gross appearance variations of an individual, for example, walking while carrying a ball or a backpack. We introduce a novel, spatiotemporal Shape Variation-Based Frieze Pattern (SVB frieze pattern) representation for gait, which captures motion information over time. The SVB frieze pattern represents normalized frame difference over gait cycles. Rows/columns of the vertical/horizontal SVB frieze pattern contain motion variation information augmented by key frame information with body shape. A temporal symmetry map of gait patterns is also constructed and combined with vertical/horizontal SVB frieze patterns for measuring the dissimilarity between gait sequences. Experimental results show that our algorithm improves gait recognition performance on sequences with and without gross differences in silhouette shape. We demonstrate superior performance of this computational framework over previous algorithms using shape cues alone on both CMU MoBo and UoS HumanID gait databases.
\end{abstract}

\section{Introduction}

Human gait recognition starts from the observation that an individual's walking style is unique and can be used for human identification. Gait recognition involves visual cue extraction and classification to identify individual walking characteristics. Gait is easier to perceive from a long distance than other biometrics like face and fingerprint. This makes gait a more attractive biometric for human identification from a far. However, gait features have a high intra-personal variation in shape influenced by external conditions like footwear, clothing and load carrying. The variation of gaits is also influenced by mood, ground surface condition and time difference. We therefore need to take both shape and motion variations into consideration for robust gait recognition.

Previous work over many years has achieved significant performance on several well known test data sets, such as Carnegie Mellon University's MoBo database [12], University of Southampton's HumanID image database [13], and University of South Florida's HumanID gait challenge database [7]. Previous work has also studied the effects of external conditions on the recognition rate. However, state of the art algorithms using shape cues alone show weakness to gross appearance variations of an individual.

In this paper, we propose a new spatiotemporal pattern that represents gait shape variation information and the relation between temporally separated gait motions based on sequences of body silhouettes. Our algorithm shows robust and improved test results over previous algorithms using shape cues on test sequences containing significant changes in an individual's body shape.

\section{Previous work on Gait recognition}

There are basically two different types of gait recognition algorithms: those that primarily use motion information and those that rely more on shape. The first class of methods includes those that use body part moments extracted from a human body silhouette [1, 2], eigengait space [3] and Hidden Markov models [4, 5, 6]. However, experimental results of these algorithms on the MoBo database [12] show worse performance compared to methods using mainly shape information [7].

Several recent studies show that shape cues in silhouette based gait recognition contribute more to the recognition results than motion cues $[8,9]$. Average silhouette is one of the most well known shape features for gait recognition [9, 10]. A collection of clustered silhouette stances with a population HMM is another shape based gait feature. The work in [11] adopts dynamics-normalized shape cues, seven stance shapes, which are not enough to represent dynamics of the entire gait. Using shape cues like body height, width, part proportions combined with motion cues like stride length and the amount of arm swing provides improved results [14].

Even though shape-based cues empirically give good recognition results [7], it has been observed that body shape cues work poorly on sequences like 'Walk with ball' of the 

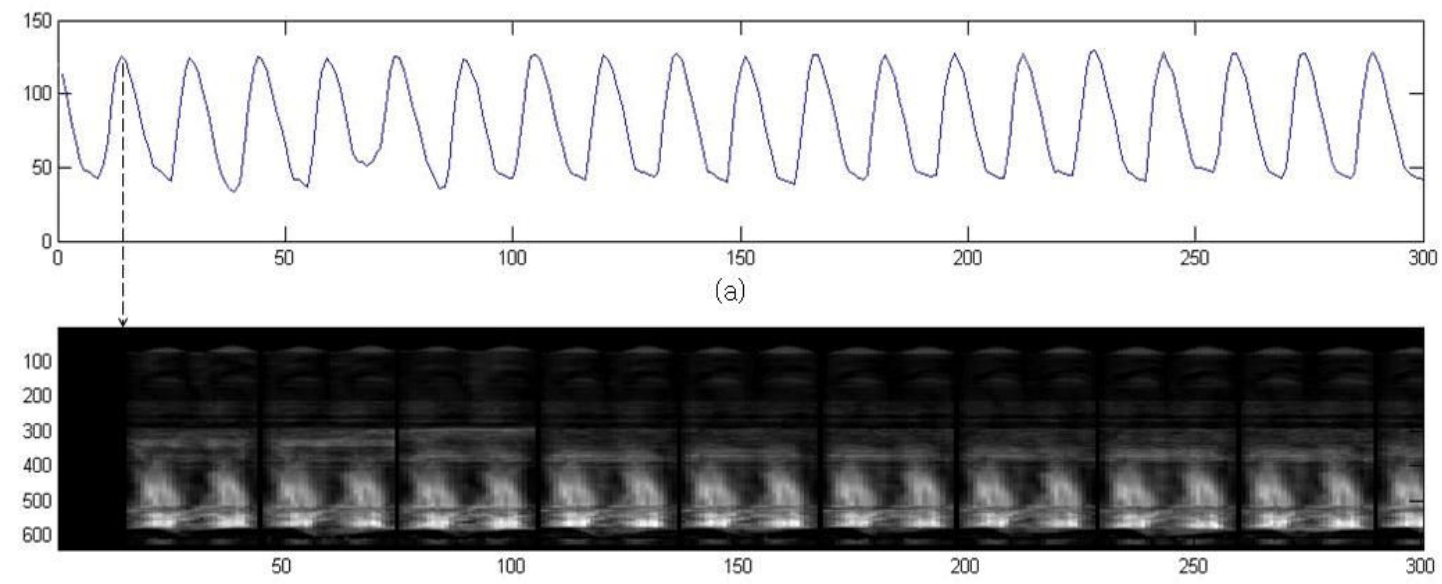

(b)

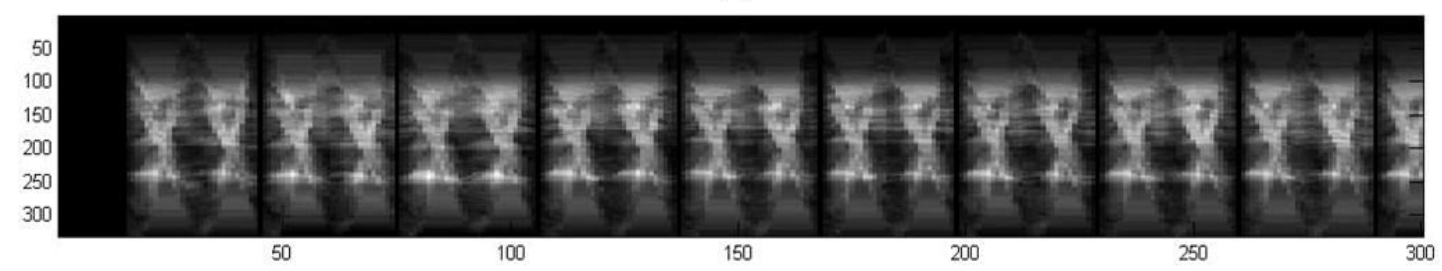

(c)

Figure 1: (a) Width variation of lower body silhouette. The lower body portion of each aligned silhouette is projected perpendicular to the horizontal axis and the width is obtained. (b) Horizontal SVB frieze pattern extracted automatically.

(c) Vertical SVB frieze pattern extracted automatically.

MoBo gait database, which exhibits significant shape change between gallery and probe silhouette for the same person [8]. This shows that we can not ignore motion information for gait recognition.

Spatiotemporal pattern matching for gait recognition is an interesting approach, because they contain both shape and temporal variation information inside the pattern, converting the gait recognition problem into a pattern recognition problem. A self similarity plot is one example of these patterns for gait recognition $[17,18]$. Self-similarity plots are obtained by computing difference values of all pairs of images. In [17], a normalized self-similarity plot for each person is used for recognition. Their work shows that shape variation information has the ability to discriminate between individual human gaits that have large variance in the real world. However, their test results are worse than other silhouette based algorithms. This is because the self similarity plot sums up all difference values over the whole non-rigid human body, losing the detailed local motion information.

Frieze patterns are another spatiotemporal pattern proposed for gait recognition [15, 16]. By projecting (summing) silhouette images along the horizontal and vertical axis respectively, a repeating spatiotemporal frieze pattern is computed over time. Frieze patterns, however, contain a shape component that is sensitive to gross appearance variations of an individual, for example, walking while carrying a ball or a backpack.

We hypothesize that appropriate shape cues combined with motion cues will give improved recognition results on sequences where the same person has different appearances, and that spatiotemporal patterns are a good tool for this purpose. We develop a novel Shape Variance-Based frieze pattern (SVB frieze pattern) representing self similarity of each row/column of a silhouette. Figure 1 (b) and (c) show $n$ examples of the SVB frieze pattern of a gait sequence.

\section{Shape Variance-Based Frieze pattern}

The algorithm for extracting an SVB frieze pattern from silhouette images of gait sequence mainly consists of two parts. First we need to extract key frames for each gait cycle. We define one gait cycle as the period starting from a double support stance frames with left foot forward to the next. To do this, we seek reliable detection of frames occurring at the same relative offset within each gait cycle (for example, double support stance frames with left foot forward). Secondly, difference frames based on subtracting these key frames from silhouettes at other times are calculated and the SVB frieze pattern is computed based on these difference frames. Figure 2 shows this process over one cycle. 


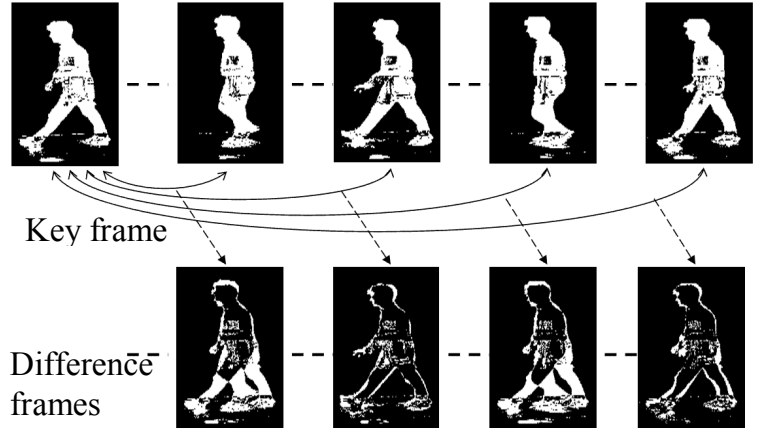

Figure 2: Difference frame calculation between key frame and a series of subsequent frames.

\subsection{Key frame selection}

An SVB frieze pattern is a self similarity cue computed from the body silhouette, using a key frame as a reference frame to calculate self similarity over each walking cycle. We define the key frame as the starting frame of one walking cycle, which is one of the two double-support positions (two feet on the ground). Each walking cycle starts from the key frame and ends before the next key frame. First, we align all silhouette images by center of mass. To find the start point of each cycle, we project the lower body portion of each aligned silhouette perpendicular to the horizontal axis and obtain its width. Figure 1 (a) is the plot of this width over time. From this width we can robustly determine double-support key frames by detecting local maxima over time. However, there are two different types (left leg front and right leg front) of double-support positions, and it is difficult to tell which peak is for which. If camera viewing angle is not exactly plane-parallel and fronto-parallel, these two different double-support positions will create different SVB frieze patterns. Therefore, detecting the two different types is important. Among the six CMU MoBo database [12] viewing angle conditions, side view sequences are used for our experiments. This provides an easy case because the camera views the human body from the upper left side and, as a result, the left foot is always located lower in its double-support frame. UoS [13] or USF test sets [7] are shot from longer distances than MoBo database and are almost plane-parallel and fronto-parallel, so left or right foot-forward can be ignored, or else features for both left/right conditions can be extracted simultaneously and classified separately, as what was done in [17].

After the key frame is obtained, a series of difference frames $D(x, y, t)$ are computed using a minimum absolute correlation method with small search region inside $r$, similar to [17].

$$
\begin{aligned}
(d x, d y)=\underset{|d x|<r,|d y|<r}{\arg \min } \sum_{x} \sum_{y} \mid I_{k e y}\left(x, y, t_{k e y}\right) & -I(x+d x, y+d y, t) \mid
\end{aligned}
$$

$D(x, y, t)=\left|I_{k e y}\left(x, y, t_{k e y}\right)-I(x+d x, y+d y, t)\right|$

$I_{k e y}\left(x, y, t_{k e y}\right)$ is a key frame, $(\mathrm{dx}, \mathrm{dy})$ is the offset for minimum frame difference and $I(x, y, t)$ is the frame at time $t$ inside a given cycle. Figure 2 shows the process of computing difference frames based on a key frame.

\subsection{SVB frieze pattern extraction}

SVB frieze patterns can be obtained by projecting pixel values of difference frames along horizontal or vertical axes in exactly the same way as the original frieze pattern method in [15]. Each 2D difference frame is converted by the projection into 1D summed "energy" values. The SVB frieze pattern is obtained by interpreting these energy values over time.

$$
\begin{aligned}
& F P_{h}(y, t)=\sum_{x} D(x, y, t) \\
& F P_{v}(x, t)=\sum_{y} D(x, y, t)
\end{aligned}
$$

Figure 1 (b) shows a horizontal SVB frieze pattern. The $\mathrm{X}$-axis is time and $\mathrm{Y}$-axis is column-axis of the original body silhouette image. Figure 1 (c) is a vertical SVB frieze pattern. The $\mathrm{Y}$-axis is now the row-axis of the original body silhouette image. Each column of a SVB frieze pattern at time $t$ represents the difference frame at time $t$.

All corresponding columns of key frames in the SVB frieze pattern should have zero values, which is the characteristic of a self similarity plot. Along the time axis, the SVB frieze pattern captures the variation of silhouette shape based on each key frame. From the normalized and aligned SVB frieze pattern, an average SVB frieze pattern unit is extracted, which is used to represent the shape variation characteristic of each subject.
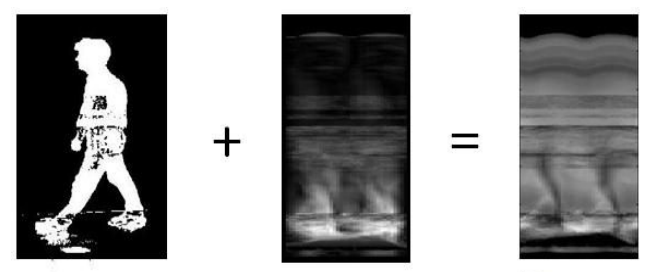

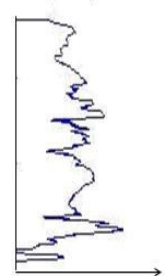

(a)

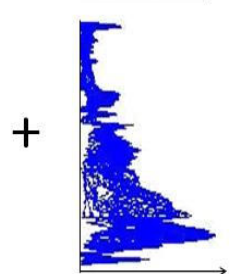

(b)

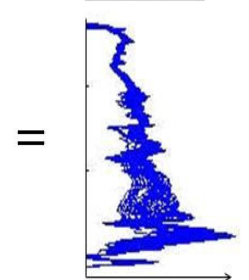

(c)
Figure 3: (a) key frame. (b) SVB frieze pattern. (c) Original frieze pattern [15] obtained by adding horizontally projected $1 \mathrm{D}$ energy value to each columns of SVB frieze pattern 


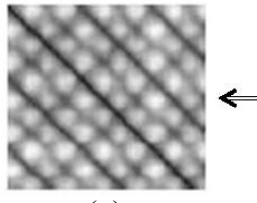

(a)

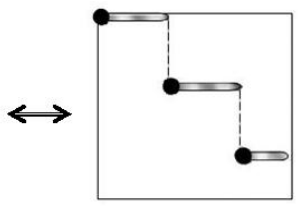

(d)

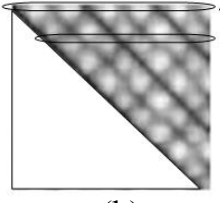

(b)
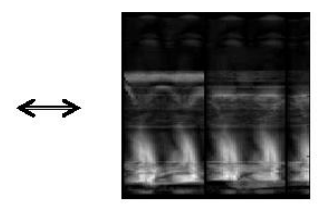

(e)
Figure 4: (a) Self-similarity plot [17] (b),(c) Redundancy elimination (d) New reference frame for each cycle which corresponds to the key frame (e) SVB frieze pattern

\subsection{SVB frieze pattern vs Frieze pattern}

SVB frieze patterns are similar to the original gait frieze patterns [15]. Both patterns are spatiotemporal plots showing variation of silhouettes over time. Since the original frieze pattern is computed over the complete silhouette image, it is sensitive to body appearance changes. Figure 3 shows that an SVB frieze pattern emphasizes the pure shape variation component of the original shape pattern. If we project each key frame to the Y-axis and add that to each column of the SVB frieze pattern, we can recover the original frieze pattern. This means that the original frieze pattern can be decomposed into a key frame representing the shape component and an SVB frieze pattern representing the motion component after subtracting the key frame.

\subsection{SVB frieze pattern vs SSP}

SVB frieze pattern is also related to the self-similarity plot [17] obtained by computing difference values of all pairs of images. Where, both $\mathrm{X}$-axis and $\mathrm{Y}$-axis are time axes representing image frames. With this characteristic, all point values along the diagonal are zero, and the lower triangle below the diagonal is an exact reflection of the upper triangle (Figure 4 (b)). Furthermore, each row of the upper triangle is a linear transformation of the first row, which means all values of a specific row can be obtained by linear combinations of values of the first row (figure 4 (C)). We can conclude that, with any arbitrary row, all other values of a self similarity plot can be recomputed. Figure 4 (C) shows one selected row that contains the same information as the whole self similarity plot (Figure 4 (a)). The black point represents the reference frame for that row (Figure 4 (c), (d)).

We can decompose each row into several small rows
(Figure $4(\mathrm{~d})$ ) for each walking cycle starting from the diagonal line. Now each small row has its own reference key frame. These key frames correspond to the key frames of each SVB frieze pattern cycle. Likewise, if we decompose each small row vertically, we can obtain the horizontal SVB frieze pattern (Figure 4 (e)). In other words, if we project horizontal SVB frieze patterns along the X-axis, we can obtain one row of the self similarity plot, from which the entire self similarity plot can be recovered.

We conclude that SVB frieze pattern is a type of self similarity plot and each row of the horizontal SVB frieze pattern represents self similarity values of the corresponding row of a silhouette image.

\section{Classification}

\subsection{Symmetry map of frieze pattern}

One gait cycle consists of two translation-symmetric half cycles. These two half cycles have almost the same pattern and period. Half of a single unit frieze pattern is nearly a shifted version of the second half, representing a temporal translation symmetry. However, it may not always be an exact symmetry and not an exact frieze pattern. Some people could swing one arm but not the other arm or a step on one side could be longer than the other. Different amounts of symmetry will be observed for different people, which can be used as a feature for recognition. The original frieze pattern approach [15] introduced the use of symmetry distance to make better decisions for classification.

In this paper, we use a symmetry map obtained from SVB frieze patterns. By computing the difference between two half motion cycles of SVB frieze patterns we can obtain a symmetry map. Figure 6 shows the symmetry maps for different individuals in the MoBo database. Each person has a different symmetry map and it is an additional shape variance-based feature for gait recognition used in our algorithm. High values of the symmetry map represent highly non-symmetric motion of the corresponding spatiotemporal region. In other words, the symmetry map not only gives a measure of gait dynamics symmetry, but also quantifies which spatiotemporal point of the gait motion has a non-symmetric nature.

Note that although we obtain a symmetry map from an SVB frieze pattern, it provides us new information about gait motion. Classification only with SVB frieze patterns matches the entire gait cycle of one sequence with the corresponding cycle of other sequences. It is an inter-cycle cue where the relation between different gait cycles is a concern. In contrast, the symmetry map represents intra-cycle correlation, which tells how two half cycles in a gait sequence are related to each other. 


\subsection{Cost function for matching}

We obtain four cues from each gait sequence: horizontal \& vertical SVB frieze pattern and horizontal \& vertical symmetry map. We then define and compute the following distance values from the $i$ 'th gallery to $j$ 'th probe along all four feature dimensions:

$$
\begin{aligned}
& \phi_{F P_{h}}=\left|F P_{h}^{i}-F P_{h}^{j}\right| \\
& \phi_{F P_{v}}=\left|F P_{v}^{i}-F P_{v}^{j}\right| \\
& \phi_{S M_{h}}=\left|S M_{h}^{i}-S M_{h}^{j}\right| \\
& \phi_{S M_{v}}=\left|S M_{v}^{i}-S M_{v}^{j}\right|
\end{aligned}
$$

Where $F P_{h}$ and $F P_{v}$ represent horizontal and vertical SVB frieze pattern. $S M_{h}$ and $S M_{v}$ represent the symmetry map for the horizontal and vertical SVB frieze pattern respectively. A single cost function is computed by summing up all four parameterized cue distance values:

$$
C_{i j}=\alpha \times \phi_{F P_{h}}+\beta \times \phi_{F P_{v}}+\gamma \times\left(\phi_{S M_{h}}+\phi_{S M_{v}}\right)
$$

\section{Experiments}

For recognition experiments we need a training sequence (Gallery) and a test sequence (Probe). There are many gait test databases that are well organized and have different types of challenging conditions. However, few of them contain sequences with significant shape change for the same subjects between gallery and probe.

We test our algorithm using two well known gait databases, the CMU MoBo (CMU) database [12] and University of Southampton's HumanID image database [13].

\subsection{CMU MoBo database}

The CMU MoBo (CMU) database is a well-known public data set used by many previous researchers. It records 25 subjects for each of four gait types (slow walk, fast walk, ball walk, inclined walk). It is useful for comparing performance against other gait recognition algorithms. Furthermore, it has a test sequence where the subject is carrying a ball, which changes body silhouette appearance considerably. The MoBo database is suitable for checking the performance of the shape variation cue compared to the previous shape-only cues. The number of walking cycles is large, which is also appropriate for training our features. In this paper, 'slow walk', 'fast walk' and 'ball walk' types are used for both gallery and probe.

MoBo images have large and well segmented silhouette images thanks to the background information provided.
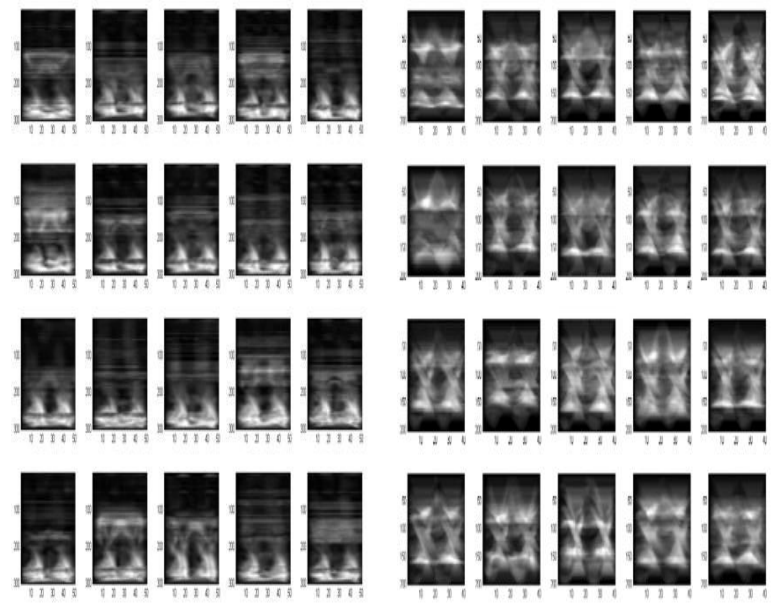

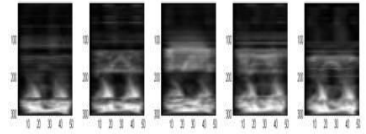

(a)

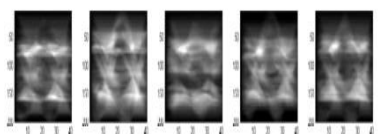

(b)
Figure 5: Normalized SVB frieze patterns of the 25 CMU MoBo subjects (a) Horizontal (b) Vertical
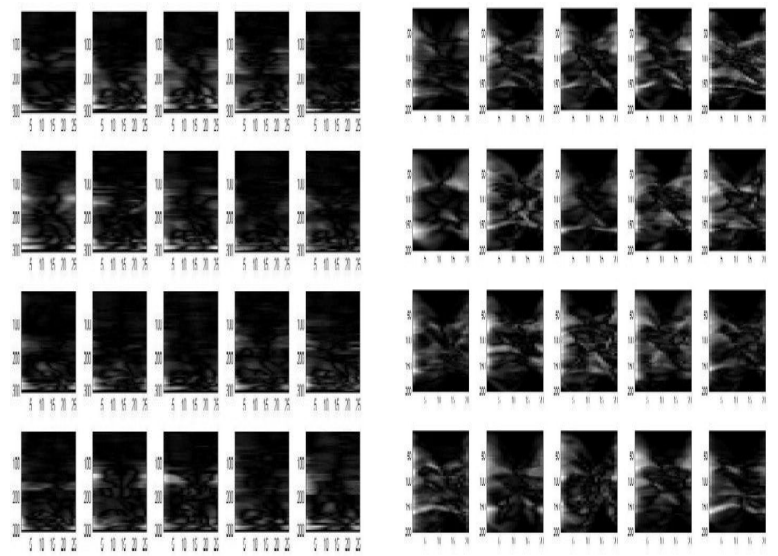

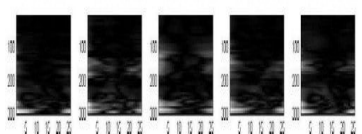

(a)
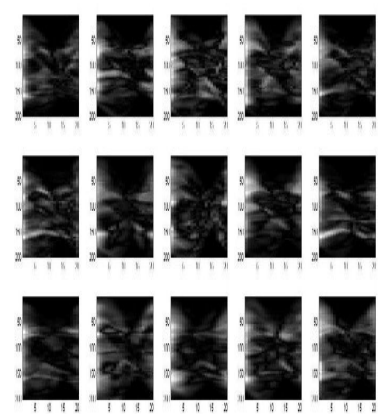

(b)
Figure 6: Symmetry maps of the $25 \mathrm{CMU}$ MoBo subjects (a) Horizontal (b) Vertical

This also gives good information for body dynamics computation. Figure 5 (a) shows 25 normalized horizontal SVB frieze pattern units and figure 5 (b) shows normalized vertical SVB frieze pattern units trained on the 'slow walk' sequence.

Table 1 shows test results comparing our algorithm against five existing methods. Previous algorithms show high recognition rates for the first five probes where shape variations between gallery and probe sequences are relatively small. 


\begin{tabular}{|c|c|c|c|c|c|c|}
\hline & $\begin{array}{c}\text { CMU } \\
{[14]}\end{array}$ & $\begin{array}{c}\text { UMD } \\
{[8]}\end{array}$ & $\begin{array}{c}\text { MIT } \\
{[1]}\end{array}$ & $\begin{array}{c}\text { Frieze } \\
{[15]^{*}}\end{array}$ & $\begin{array}{c}\text { SSP } \\
{[17]}\end{array}$ & $\begin{array}{c}\text { SVB } \\
\text { frieze }\end{array}$ \\
\hline S/S & $100 \%$ & $100 \%$ & $100 \%$ & $100 \%$ & $100 \%$ & $100 \%$ \\
\hline F/F & - & $100 \%$ & - & $100 \%$ & $100 \%$ & $100 \%$ \\
\hline B/B & - & $92 \%$ & - & $100 \%$ & - & $100 \%$ \\
\hline S/F & $76 \%$ & $80 \%$ & $64 \%$ & $100 \%$ & $54 \%$ & $82 \%$ \\
\hline F/S & - & $84 \%$ & - & $84 \%$ & $32 \%$ & $80 \%$ \\
\hline S/B & $92 \%$ & $48 \%$ & $50 \%$ & $81 \%$ & - & $77 \%$ \\
\hline B/S & - & $68 \%$ & - & $50 \%$ & - & $89 \%$ \\
\hline F/B & - & $48 \%$ & - & $50 \%$ & - & $61 \%$ \\
\hline B/F & - & $48 \%$ & - & $50 \%$ & - & $73 \%$ \\
\hline
\end{tabular}

Table 1: CMU MoBo recognition results with $\mathrm{S}$ (slow walk), $\mathrm{F}$ (fast walk) and B (ball walk) sequences. S/F represents Gallery S and Probe F.

*Test results for frieze pattern are based on internal experiments.

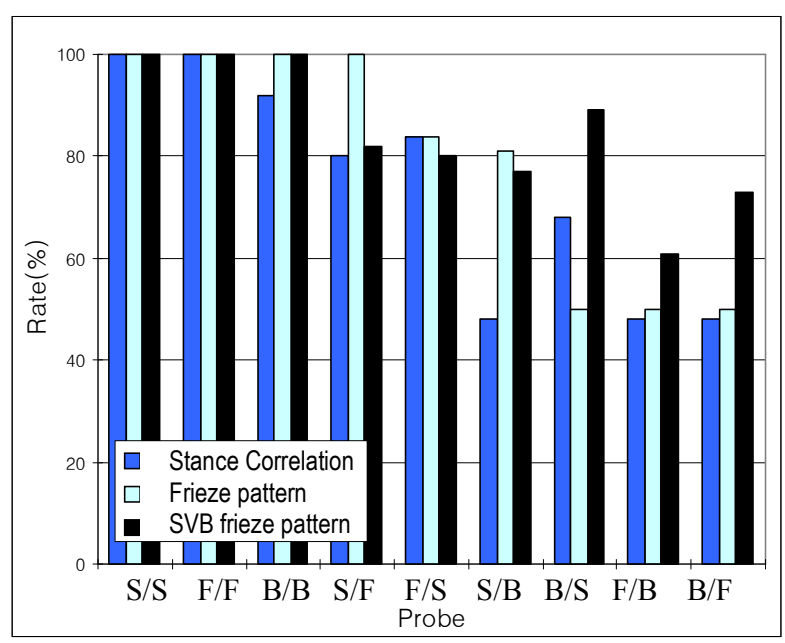

Figure 7: Top rank result of MoBo database. Last four test results for 'ball walk' sequences are notably improved.

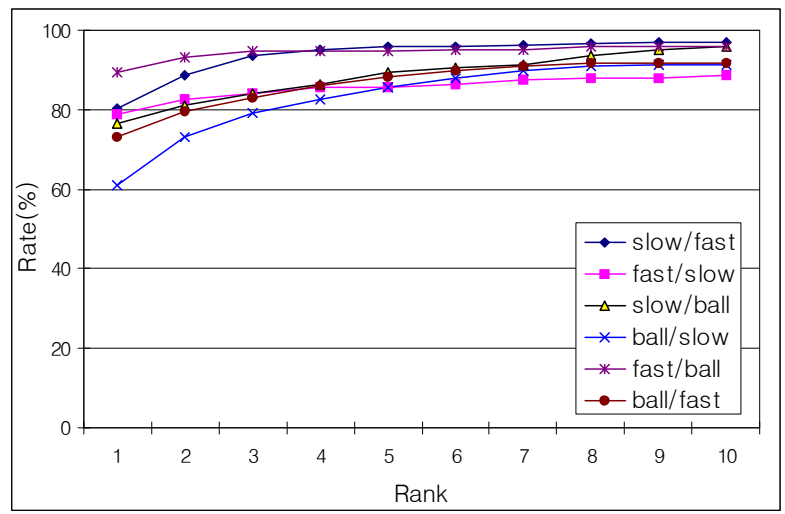

Figure 8: Cumulative Match Score (CMS) of six gallery/probe combination test results using proposed SVB frieze pattern on the CMU MoBo database.
However, for the last four test results including 'ball walk' sequence, they have relatively low recognition rates, which tells us that their algorithms are not robust to appearance changes. In contrast, our algorithm yields good performance across all types of gallery/probe combinations showing best or second best classification rate. Especially, the performance of our algorithm on the 'ball walk' related tests is better than any previous algorithm. Figure 7 compares three selected algorithms across all types of test result.

\subsection{UoS HumanID image database}

We also tested our algorithm with University of Southampton's HumanID image database [13]. It shows 116 people under different conditions. Silhouettes are clean and large enough to show detailed human body motion. However, it does not provide multiple sequences for the same person. To check the robustness of our gait recognition algorithm, multiple test sequences of the same person having different silhouette appearance conditions are necessary.

We simulated new sequences with gross changes in body shape by adding a square blob on the back of the body (Probe $\mathrm{B}$ ) and by dilating the torso part of the silhouette (Probe $\mathrm{C} 1 \sim \mathrm{C} 8$ ) by different disk elements of radius from 3 to 17. Figure 9 (a) shows selected single frames for each sequence. Horizontal body center and vertical torso part center values are used to place the newly added square blobs (Probe B) at every frame.

Probe A represents both training and testing with original database images. The first 5 gait cycles for each sequence are used for training and all other cycles are used for testing. Probes $\mathrm{B}$ and $\mathrm{C}$ represent training with the first 5 cycles of each sequence of original data and testing with other cycles after adding a blob or dilating the sequence. Probe B looks

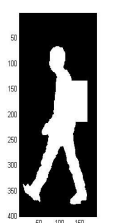

B

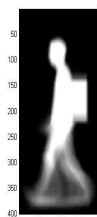

B

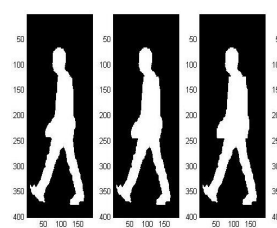

C1

$\mathrm{C} 2$

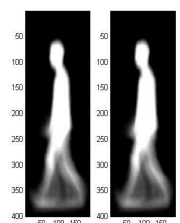

$\mathrm{C} 1$
$\mathrm{C} 2$
$\mathrm{C} 3$<smiles>[Li]</smiles>

(a)

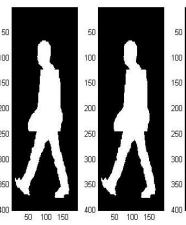

C5

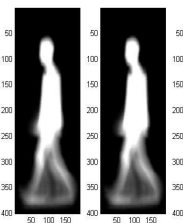

$\mathrm{C} 4$

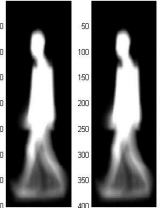

C5
C6

$\mathrm{C} 8$
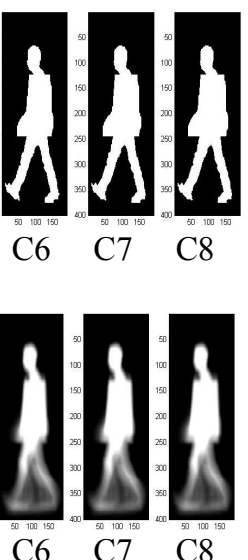

(b)

Figure 9: (a) sample frame from probe B and C (b) Average silhouette of probe $\mathrm{B}$ and $\mathrm{C}$ 
like carrying a backpack, and changes a small part of the human body by adding a rigid object that does not reflect human body local dynamics. Only global periodic motion affects the location of the added blob. Probe $\mathrm{C}$ has 8 gradually dilated sequences that look like wearing heavy clothes. Previous work [11] using stance shape cues also reports an algorithm robust to overall dilation or erosion of silhouette. However, their concern is recovering clean and consistent silhouettes from those contaminated by noise or background clutter. Real intra-personal shape variation due to clothing can have partial changes or non-linear characteristics. The head part could be changed by wearing a cap or only body parts could be changed by wearing a heavy coat. No eigen-shape is preserved in this case, and only eigen shape variation can be kept, which is represented by the SVB frieze pattern.

\begin{tabular}{|c|c|c|c|}
\hline & $\begin{array}{c}\text { Avg. } \\
\text { silhouette [9] }\end{array}$ & $\begin{array}{c}\text { Frieze } \\
\text { Pattern [15] }\end{array}$ & $\begin{array}{c}\text { SVB Frieze } \\
\text { Pattern }\end{array}$ \\
\hline A & $100 \%$ & $96 \%$ & $84 \%$ \\
\hline B & $94 \%$ & $78 \%$ & $82 \%$ \\
\hline C1 & $100 \%$ & $95 \%$ & $82 \%$ \\
\hline C2 & $96 \%$ & $82 \%$ & $78 \%$ \\
\hline C3 & $53 \%$ & $56 \%$ & $74 \%$ \\
\hline C4 & $35 \%$ & $47 \%$ & $73 \%$ \\
\hline C5 & $28 \%$ & $41 \%$ & $73 \%$ \\
\hline C6 & $22 \%$ & $39 \%$ & $73 \%$ \\
\hline C7 & $22 \%$ & $38 \%$ & $72 \%$ \\
\hline C8 & $18 \%$ & $37 \%$ & $72 \%$ \\
\hline
\end{tabular}

Table 2: UoS HumanID database recognition results. Original silhouette A is used for gallery. A, B and $\mathrm{C} 1 \sim \mathrm{C} 8$ are used for probe.

Figure 9 (b) shows corresponding average silhouettes, which are basic shape cues used in many previous works [9, 10,11]. Average silhouette, original frieze pattern and SVB frieze pattern are compared in Figure 10. Absolute difference is used for classification of each feature. For test sequences with the added square blobs, all features still show high recognition rates, but the previous two features, average silhouette and frieze pattern, show larger degradation in performance than our proposed method using the SVB frieze pattern (Table 2). Our SVB frieze pattern is not affected by drastic appearance change as much as those two existing methods (Figure 10). We also can observe that the original frieze pattern still works better than average silhouette under appearance change because it also contains a component of shape variation in it. Notice that the frieze pattern has a time axis in its representation, but an average silhouette just has two spatial axes.

\section{Conclusion}

We propose a novel shape variation-based frieze pattern

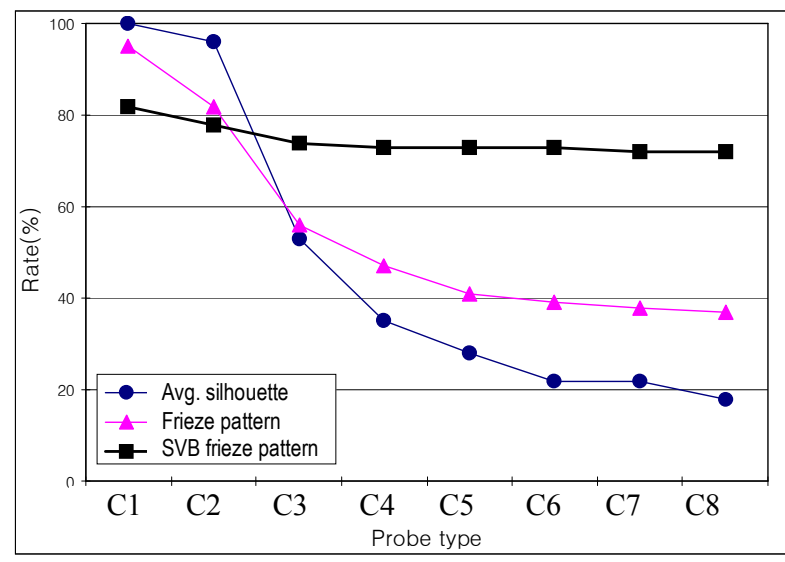

Figure 10: Test result of $\mathrm{C} 1 \sim \mathrm{C} 8$ probe sequences. SVB frieze pattern shows robust recognition result with appearance change.

representation and a symmetry map representation for gaits that capture the intra and inter-shape variations, respectively. Jointly using these features for gait recognition enhances recognition performance, especially when there is a serious silhouette appearance variation between gallery and probe sequences. We verify our algorithm experimentally and compare it with several recent published gait recognition systems on the CMU MoBo database and UoS HumanID image database. Unfortunately, not enough public data is available with multiple sequences of significant appearance change for the same person. Therefore we simulated appearance change in the UoS silhouette data. These new data sets enable us to show the strength of our algorithm.

Our test results using only key frames for human identification show how the shape component contributes to gait recognition. Table 3 shows the recognition rates using CMU MoBo 'Slow' and 'Fast' sequences, which has low appearance change.

\begin{tabular}{|c|c|c|c|c|}
\hline & $\begin{array}{c}\text { Slow } \\
\text { /Slow }\end{array}$ & $\begin{array}{c}\text { Fast } \\
/ \text { Fast }\end{array}$ & $\begin{array}{c}\text { Slow } \\
/ \text { Fast }\end{array}$ & $\begin{array}{c}\text { Fast } \\
/ \text { Slow }\end{array}$ \\
\hline $\begin{array}{c}\text { Key } \\
\text { frame }\end{array}$ & $99 \%$ & $96 \%$ & $90 \%$ & $86 \%$ \\
\hline
\end{tabular}

Table 3: Identification result with MoBo database using only key frames

A key frame is a single silhouette image that does not contain any gait dynamics. So we can not consider an algorithm based only on key frames gait recognition. It is nothing but body shape recognition. We can conclude from the good recognition results (Table 3 ) using only key frames that if we include this body shape information inside the feature used for gait recognition, test results will be significantly helped by the shape recognition information. Indeed, imagine that all frames of a gait sequence are 
randomly shuffled. Now no more gait motion exists with these shuffled frames. However, the average silhouette or similar average stance features of the original sequence and new shuffled sequence will be exactly the same. Normalized dynamics algorithms [11] will suffer this problem less, however their seven stance-shapes still are an average of clustered silhouettes for periods shorter than one gait cycle.

Although shape cues could be a good component for gait recognition, gait is a kind of body activity and gait recognition starts from the observation that gait dynamics can be treated as a biometric. Our SVB frieze pattern incorporates both key frame shape and motion variation information with respect to key frame values. The key frame plays a role of DC component of this variation, so as to keep the body structure and define its distribution. In other words, the key frame is a human body shape model over one gait cycle on which motion of gait is superimposed.

SVB frieze patterns improve overall recognition performance under gross changes in body shape, however they are not the best performing algorithm if body shape does not change so much (Table 1). To achieve better performance across all types of sequences, we propose to combine shape cues with SVB frieze patterns in a hierarchical system for gait recognition in our future work.

Instead of the absolute distance we used for classification, other distance measures like cross correlation or sum of squared distance can be used. It is also possible to use Linear Discriminant Analysis (LDA) with absolute distance to improve recognition rates [11].

\section{Acknowledgment}

This research is a class project for "Computational Symmetry" CSE598B taught by Dr. Yanxi Liu in FALL 2006, Computer Science \& Eng. Dept. Penn State University.

\section{References}

[1] L. Lee and W. Grimson, "Gait Analysis for Recognition and Classification," Proc. Int'l Conf. Automatic Face and Gesture Recognition, pp. 155-162, 2002.

[2] L. Lee, G. Dalley, and K. Tieu, "Learning Pedestrian Models for Silhouette Refinement," Proc. Int'l Conf. Computer Vision, 2003.

[3] C. BenAbdelkader, R. Cutler, and L. Davis, "Motion-Based Recognition of People in Eigengait Space," Proc. Int'l Conf. Automatic Face and Gesture Recognition, pp. 267-272, 2002.

[4] A. Kale, N. Cuntoor, and R. Chellappa, "A Framework for Activity Specific Human Identification," Proc. Int'l Conf. Acoustics, Speech, and Signal Processing, 2002.

[5] A. Sunderesan, A.K.R. Chowdhury, and R. Chellappa, "A Hidden Markov Model Based Framework for Recognition of Humans from Gait Sequences,” Proc. IEEE Int'l Conf. Image Processing, 2003.

[6] A. Kale, A. Sundaresan, A.N. Rajagopalan, N.P. Cuntoor, A.K.Roy-Chowdhury, V. Kru" ger, and R. Chellappa,
"Identification of Humans Using Gait," IEEE Trans. Image processing, vol. 13, NO. 9, Sep. 2004

[7] S. Sarkar, P.J. Phillips, Z. Liu, I.R. Vega, P. Grother, and K.W. Bowyer, "The Humanid Gait Challenge Problem: Data Sets, Performance, and Analysis,"IEEE Trans. Pattern Analysis and Machine Intelligence, vol. 27, no. 2, pp. 162-177, Feb. 2005.

[8] A. Veeraraghavan, A. R. Chowdhury, and R. Chellappa, "Role of shape and kinematics in human movement analysis," in Proc. IEEE Conf. Computer Vision Pattern Recognition, Washington DC, Jun. 2004.

[9] G.V. Veres, L. Gordon, J.N. Carter, M.S. Nixon, "What Image Information Is Important in Silhouette-Based Gait Recognition?", 2004 IEEE Computer Society Conference on Computer Vision and Pattern Recognition (CVPR'04) Volume 2 pp. 776-782

[10] J. Han and B. Bhanu, "Statistical Feature Fusion for Gait-Based Human Recognition," Proc. IEEE Conf. Computer Vision and Pattern Recognition, vol. 2, pp. 842-847, June 2004.

[11] Zongyi Liu, Sudeep Sarkar, "Improved Gait Recognition by Gait Dynamics Normalization", IEEE Transactions on Pattern Analysis and Machine Intelligence, vol. 28, no. 6, pp. 863-876, June 2006.

[12] R. Gross and J. Shi, "The CMU Motion of Body (MoBo) Database,"Technical Report CMU-RI-TR-01-18, Robotics Inst., Carnegie Mellon Univ., June 2001.

[13] J. D. Shutler, M. G. Grant, M. S. Nixon, and J. N. Carter "On a Large Sequence-Based Human Gait Database", Proc. 4th International Conference on Recent Advances in Soft Computing, Nottingham (UK), pp 66-71, 2002

[14] R.Collins, R.Gross and J.Shi, "Silhouette-based Human Identification from Body Shape and Gait," Intl' Conference on Face and Gesture, October 2002, pp.351-356.

[15] Y.Liu, R.Collins and Y.Tsin, "Gait Sequence Analysis using Frieze Patterns," European Conference on Computer Vision, Copenhagen, May 2002, pp.657-671. Also, Technical Report CMU-RI-TR-01-38, Robotics Institute, Carnegie Mellon University, December, 2001.

[16] Y. Liu, R. Collins, and Y. Tsin, "A Computational Model for Periodic Pattern Perception Based on Frieze and Wallpaper Groups", IEEE Transactions on Pattern Analysis and Machine Intelligence, Vol. 26, No. 3, March, 2004, pp. 354 371

[17] Chiraz BenAbdelkader, Ross G. Cutler, and Larry S. Davis , "Gait Recognition Using Image Self-Similarity", EURASIP Journal on Applied Signal Processing, Volume 2004 (2004), Issue 4, Pages 572-585

[18] R. G. Cutler and L. S. Davis, "Robust real-time periodic motion detection, analysis and applications," IEEE Trans. on Pattern Analysis and Machine Intelligence, vol. 22, no. 8, pp. 781-796, 2000. 